\title{
Influence of Storage Condition on Flexure Properties of Nano-Composite
}

\section{Dalia A Abuelenain*}

Department of Restorative Dentistry, Faculty of Dentistry, King Abdulaziz University, Jeddah, Saudi Arabia

*Corresponding author: Dalia A Abuelenain, Department of Restorative Dentistry, Faculty of Dentistry, King Abdulaziz University, Jeddah, Saudi Arabia, Tel: 00966 505679543, E-mail: dabualenain@kau.edu.sa

Citation: Dalia A Abuelenain (2017) Influence of Storage Condition on Flexure Properties of Nano-Composite. J Dent Oral Care Med 3(1): 105. doi: 10.15744/2454-3276.3.105

Received Date: April 13, 2017 Accepted Date: May 14, 2017 Published Date: May 23, 2017

\begin{abstract}
Objective: To evaluate the flexure strength and flexure modulus of nano-filled dental composite after different storage conditions.

Methods: Two nano-composite materials were investigated (Filtek Z350 and Tetric N Ceram). Composite bars ( $\mathrm{n}=15$ ) were prepared from each material using Teflon mold with the dimensions of $(2 \times 2 \times 25 \mathrm{~mm})$. Samples from each material were randomly subdivided into three groups according to storage condition (dry, distilled water, and thermo-cycled). Flexure test were performed under a load of $2 \mathrm{KN}$ at a cross head speed of $0.25 \mathrm{~mm} / \mathrm{min}$ in a universal testing machine.

Results: There was no statistical significance difference in flexure strength and flexure modulus of the same composite material under different storage conditions ( $\mathrm{p}<0.05$ ). In addition, the flexure moduli of Filtek Z350 were significantly higher than Tetric N Ceram under all storage conditions $(\mathrm{p}<0.05)$.

Conclusion: Thermo-cycling, short-term dry or wet storage before mechanical testing did not influence the flexure properties of nano- composite.
\end{abstract}

Keywords: Nano-composite; Thermo-cycling; Flexure strength; Flexure modulus

\section{Introduction}

Resin based composite (RBC) restorations are widely used in dental practice. The improved physical and mechanical properties of $\mathrm{RBC}$, the good esthetics, and the ease of chair side application in addition to acceptable durability of these restorations have led to its high popularity in recent decades. Durability of dental composite restorations is influenced by multiple factors, some are related to materials composition such as resin matrix, filler type and filler loading and others are related to the technique and surrounding environment in which the material is functioning, and recurrent decay could be an additional contributing factor to composite failure [1-8].

Dental composite restorations in the oral cavity are subjected to temperature variations during function due to consumption of cold and hot foods and beverages [9]. However, in literatures, there are variable techniques of sample storage before mechanical testing. Composite test samples may be stored at room temperature, body temperature, or thermo-cycled to represent temperature variation in the oral cavity $[7-15,17]$. In addition, water was used as an aging solution in several studies to mimic the wet environment in the oral cavity $[4,18-20]$. Also, variability exists in the storage medium in different literatures. Prepared samples may be stored dry in closed containers or kept wet in distilled water, artificial saliva or different types of liquids [10-14]. These variations may influence material behavior and mechanical test results.

Some of these studies showed that water storage and thermo-cycling before testing may have a deleterious effect on mechanical behavior of resin materials [5,21,22], while others show either no influence of these variables [8] or even improved mechanical properties after thermo-cycling [9].

Testing of the same commercially available composite material by different investigators under different testing conditions, has resulted in different mechanical behavior of the same material. This can be attributed to many factors such as techniques of sample preparation, testing variables, testing environment and storage conditions before testing. Interaction between environmental conditions and inherent material properties may influence materials behavior, and testing dental materials under different environmental conditions will show different responses. How does a different storage condition influence the material behavior during testing is still an area of conflict, and selecting a dry or wet storage medium for test samples was not justified by some researchers in several investigations. 
This makes the selection of the ideal storage condition to achieve standardized results, a controversial issue.

In order to minimize testing variables, in vitro testing of RBC should mimic the oral environment as much as permitted, in order to predict the mechanical performance of these materials under different clinically relevant conditions. However, close reproduction of oral environment during storage of test samples is challenging.

\section{Aim}

Aim of the present study was to investigate the influence of dry and wet storage and thermo-cycling on flexure strength and flexure modulus of nano-composite materials.

\section{Null hypothesis:}

I. There is no significant difference between tested nano-composite materials with regard to their flexure properties.

II. Storage of composite samples in dry environment, wet in distilled water or thermo-cycling of the samples, before mechanical testing, will not influence flexure strength and flexure modulus of the tested composite.

\section{Materials and Methods}

Two nano-hybrid composite materials were used in the study, technical details are presented in table 1.

\begin{tabular}{|c|c|c|c|c|}
\hline Composite & Manufacturer & Type & Resin Matrix & Filler \\
\hline $\begin{array}{c}\text { Filtek } \\
\text { Z350 }\end{array}$ & $\begin{array}{c}\text { 3M ESPE, St. } \\
\text { Paul, Mn, USA }\end{array}$ & Nanocomposite & $\begin{array}{c}\text { BisGMA, } \\
\text { UDMA, TEGDMA } \\
\text { and BisEMA }\end{array}$ & $\begin{array}{c}\text { Zirconia/silica and silica } \\
\text { nanoparticle; }\end{array}$ \\
\hline $\begin{array}{c}\text { Tetric N } \\
\text { Ceram }\end{array}$ & $\begin{array}{c}\text { Ivoclar } \\
\text { Vivadent }\end{array}$ & Nanocomposite & $\begin{array}{c}\text { BisGMA, } \\
\text { UDMA, TEGDMA } \\
\text { and BisEMA }\end{array}$ & $\begin{array}{c}\text { Barium glass, ytterbium } \\
\text { trifluoride, mixed oxide, } \\
\text { silicon dioxide }\end{array}$ \\
\hline
\end{tabular}

Abbreviations: Urethane dimethacrylate (UDMA), bisphenol A glycidyl methacrylate (Bis GMA), Triethyleneglycol dimethacrylate (TEGDMA), Ethoxylatedbisphenol A dimethacrylate (BisEMA)

Table 1: Technical details of the composite materials used in the study

Fifteen bar shaped samples were prepared from each composite material using a Teflon mold $\left(2 \times 2 \times 25 \mathrm{~mm}^{2}\right)$ according to ISO 4049 and sandwiched between two celluloid strips and glass slides [23]. Samples were cured in four overlapping cycles 20 seconds each from both sides using Dentamirica, QTH curing light ((LITEX 682 Dentamirica, USA) with 7 mm curing tip at an output irradiance of $700 \mathrm{~mW} / \mathrm{cm}^{2}$; verified between samples using a radiometer (LITEX 682 Dentamirica, USA). Excess material was removed using abrasive paper (320 grit). Prepared samples were randomly divided into three groups ( $n=5)$ according to the storage condition. Group 1(Dry): stored dry in closed polyethylene container at room temperature $\left(22^{\circ} \mathrm{C}\right)$ for 7 days and used as a control. Group 2 (Wet): stored in distilled water for 7 days at room temperature. Group 3(TC): samples were subjected to 5000 cycles of thermo-cycling in distilled water at 5 and $55^{\circ} \mathrm{C}$ for 30 s each.

After storage, dimensions of each specimen were determined with a micrometer accurate to $0.001 \mathrm{~mm}$. Samples were subjected to three-point flexure test using a universal testing machine (Instron 5944, USA) with a span of $20 \mathrm{~mm}$ at a cross head speed of 0.25 $\mathrm{mm} / \mathrm{min}$ and $2 \mathrm{KN}$ load.

The flexure strength $(\mathrm{MPa})$ and modulus $(\mathrm{GPa})$ were calculated using Bluehill 3 software.

\section{Statistical Analysis}

Data collected and analyzed using SPSS (Version 20.0, IBM, and New York, USA). Data were subjected to two-way ANOVA to investigate the interaction between the dependent variable (Flexure strength or flexure modulus) and the independent variables (materials and storage condition), followed by one-way ANOVA and Tukey's post-hoc tests at $\mathrm{p}=0.05$, to delineate areas of significance.

\section{Results}

Mean values and standard deviation of flexure strength and flexure modulus are presented in table 2.

Two-way ANOVA test revealed a significant influence of the material type $(\mathrm{p}=0.002)$ on the flexure strength values, however the storage conditions and the interaction between material and storage condition did not have a significant influence on flexure strength results $(\mathrm{p}>0.05)$.

On the other hand, the materials and storage condition were having a significant influence on flexure modulus ( $\mathrm{p}<0.05$ ), while the interaction between both were not showing a significant influence $(\mathrm{p}=0.05)$.

Turkey's post hoc test revealed that, there was no statistical significance difference in flexure strength and flexure modulus between different storage conditions for each type of composite material ( Filtek Z350 and Tetric N Ceram) $(\mathrm{P}>0.05)$. And there was no statistically significant difference in flexure strength between different composite materials under the same storage condition $(\mathrm{P}>0.05)$. 


\begin{tabular}{|l|c|c|c|c|c|c|}
\hline \multirow{2}{*}{ Composite } & \multicolumn{3}{c|}{ Filtek Z350 } & \multicolumn{3}{c|}{ Tetric N Ceram } \\
\cline { 2 - 7 } & Dry & Wet & TC & Dry & Wet & TC \\
\hline Flexure Strength (SD) & $122 \mathrm{~A}$ & $108 \mathrm{AB}$ & $108 \mathrm{AB}$ & $92 \mathrm{AB}$ & $95 \mathrm{AB}$ & $74 \mathrm{~B}$ \\
$\mathrm{MPa}$ & $(15.5)$ & $(21)$ & $(23)$ & $(20)$ & $(7)$ & $(7)$ \\
\hline Flexure Modulus(SD) & $9.5 \mathrm{a}$ & $9 \mathrm{a}(1)$ & $10 \mathrm{a}$ & $7 \mathrm{~b}(1)$ & $5.5 \mathrm{~b}$ & $6 \mathrm{~b}(0.5)$ \\
$\mathrm{GPa}$ & $(1)$ & & $(1)$ & & $(0.2)$ & \\
\hline
\end{tabular}

Different alphabetical letters of the same case represent significant difference between different test groups in the same raw at $\mathrm{p}=0.5$.

Table 2: Mean and slandered deviation of flexure strength (MPa) and flexure 290 modulus (GPa) of tested materials

However, there was a significant difference between flexure strength values of Filtek Z350 samples stored at dry condition and the thermo-cycled Tetric N Ceram materials ( $\mathrm{P}=0.01)$.

In addition, the flexure moduli of Filtek Z350 were significantly higher than Tetric $\mathrm{N}$ Ceram under different storage conditions $(\mathrm{P}<0.05)$.

\section{Discussion}

The variations of temperature in oral cavity when consuming hot or cold food may influence mechanical behavior of different restorative materials [8]. Temperature in oral cavity during food consumption ranges from $1^{\circ} \mathrm{C}$ to $55^{\circ} \mathrm{C}$ [9]. Thermo-cycling is a procedure performed before mechanical testing of dental materials to simulate temperature variations during eating, drinking and breathing [24,25]. In the present study, a total of 5000 cycles of thermo-cycling were applied to simulate long term aging of about 500 days in oral cavity condition [8].

Aging of dental material before testing by immersion in water either with or without thermocycling is a common protocol for evaluation of material behavior under clinically relevant conditions. It has been established in previous investigations that water content will influence mechanical properties of resin based materials $[21,22,26]$.

Water-induced degradation was attributed to two mechanisms, one mechanism via resin absorption of water which will result in matrix swelling and it will become weak and ductile [21,26,27]. However, water sorption and post-immersion resin expansion of composite resin show a wide variability in currently available composite materials [27]. The second mechanism of water degradation is through degradation of the silane interphase resulting in weakening of mechanical properties due to hydrolysis reaction that resulted in debonding of the filler particles from the resin matrix, this debonding may result in interfacial microcracks formation causing weakening of the composite material $[26,28,29]$. It has been found in previous investigation that, dehydration of the water stored samples recovered the mechanical properties, therefore, water acted as a plasticizer and when removed the strength properties were recovered [4].

Results of the current work showed that the flexure strength of nano-composite was not influenced by different storage environments tested (dry, wet in distilled water or thermocycled). However, the materials difference significantly influenced the flexure strength and flexure modulus.

Therefore, the results of the present work requires rejection of the first part of null hypothesis in which different nano-composite materials showed significant difference in flexure properties, and acceptance of the second part of null hypothesis in which the storage condition did not influence the flexure properties.

Thermo-cycling in the present work did not deteriorate flexure properties of tested material. This was in accordance with the results of a previous study, in which 2000 cycles of thermo-cycling followed by immersion in beverages, did not influence the mechanical properties of nano-filled composite compared to conventional hybrid composite [8]. However, in another study, the influence of thermo-cycling on flexure properties was material dependent, in which most of tested materials showed increased flexure modulus after thermocycling [9]. The improved mechanical properties after thermo-cycling was inferred to the thermal stresses generated during thermo-cycling process and improve the bond strength between the composite components decreasing the space between polymer chains. Thus, diffusion of water molecules into the resin material will be reduced.

Results of the present work conflicts with previous studies in which water storage for 7 days and thermo-cycling did not influence flexure strength and flexure modulus $[18,27]$. However, these results were in agreement with another investigation in which prolonged water storage influenced flexure strength of some tested materials and did not influence the flexure modulus of all tested composite materials and another investigation was confirming the present results in which thermo-cycling did not influence flexure properties of nano-filled composite [17,30].

In addition, previous work showed that the nano-clusters in nano-filled composite would influence the strength and reliability of the composite, irrespective of the storage and testing condition, which may explain the results of the current work [6,26]. 
In the present study, comparing the two composite materials at the same storage condition showed that Z350 had non-significant higher flexure strength when compared to TNC, and a statistically significant higher flexure modulus of Z350. The two composite materials used in the present study are similar in resin component and they differ in filler loading and filler type. This could explain the higher flexure properties of Filtek Z350 with $78.5 \mathrm{wt} \%$ filler loading compared to Tetric N Ceram with $63.5 \mathrm{wt} \%$ filler loading. This was in accordance with previous work, in which the flexure properties of Z350 were significantly higher than those for TNC and theses results were attributed to the differences in filler loading, composition and size [31].

Lassila., et al. found that the dehydration of test samples before testing will result in regain of the lost mechanical properties caused by the plasticizing effect of water [4]. This explains the results of the current work, in which the test samples were dehydrated after the 7 days storage period. Therefore, wet storage of test samples will not provide the intended wet environment simulation. However, in order to mimic the oral environment, the test of composite samples has to be performed to wet samples, under wet conditions.

In addition, the insignificant influence of the ageing process utilized in the present work may be inferred to the short storage time. Seven days, therefore, did not allow sufficient time for the water-degradation to take place, however, previous study's water storage time was for 1, 7, 28, 90, 180 days, in which some composite materials showed lower flexure strength after 7 days of aging, while other composite materials showed lower flexure values after 28 or 90 days of aging [7].

Therefore, for researches, if the prepared samples are to be tested within short storage period ( 7 days or less), there will be no difference in flexure behavior of the material. However, in order to mimic oral environment, materials should be stored in wet environment for longer period of time or subjected to more thermal cycles, and the testing of the samples should be performed under wet environment.

\section{Conclusion}

Within the limitations of the current work, it can be concluded that the flexure properties of different nano-composite materials are influenced by different material composition.

Short term water storage or thermo-cycling, followed by dehydration, will not influence flexure properties of nano-filled resin based composite materials.

\section{Acknowledgment}

The author would like to acknowledge the Advanced Technology Dental Research Laboratory at King Abdulaziz University, Faculty of Dentistry and Mr. Fahad Al-Sadie, for conduction of the laboratory testing of this work.

\section{References}

1. Sunbul HA, Silikas N, Watts DC (2016) Surface and bulk properties of dental resin- composites after solvent storage. Dent Mater 32 : $987-97$.

2. Porto IC, de Aguiar FH, Brandt WC, Liporoni PC (2013) Mechanical and physical properties of silorane and methacrylate-based composites. J Dent 41: 732-9.

3. Lohbauer U, Belli R, Ferracane JL (2013) Factors involved in mechanical fatigue degradation of dental resin composites. J Dent Res 92: 584-91.

4. Lassila LV, Nohrstrom T, Vallittu PK (2002) The influence of short-term water storage on the flexural properties of unidirectional glass fiber-reinforced composites. Biomaterials 23: 2221-9.

5. Drummond JL (2008) Degradation, fatigue and failure of resin dental composite materials. J Dent Res 87: 710-9.

6. Curtis AR, Palin WM, Fleming GJ, Shortall AC, Marquis PM (2009) The mechanical properties of nanofilled resin-based composites: the impact of dry and wet cyclic preloading on bi-axial flexure strength. Dent Mater 25: 188-97.

7. Stawarczyk B, Egli R, Roos M, Ozcan M, Hammerle CH (2011) The impact of in vitro aging on the mechanical and optical properties of indirect veneering composite resins. J Prosthet Dent 106: 386-98.

8. Ayatollahi MR, Yahya MY, Karimzadeh A, Nikkhooyifar M, Ayob A (2015) Effects of temperature change and beverage on mechanical and tribological properties of dental restorative composites. Mater Sci Eng C Mater Biol Appl 54: 69-75.

9. Stewardson DA, Shortall AC, Marquis PM (2010) The effect of clinically relevant thermocycling on the flexural properties of endodontic post materials. J Dent 38: 437-42.

10. Rodrigues Junior SA, Zanchi CH, Carvalho RV, Demarco FF(2007) Flexural strength and modulus of elasticity of different types of resin-based composites. Braz Oral Res 21: 16-21.

11. Leprince JG, Palin WM, Vanacker J, Sabbagh J, Devaux J, et al. (2014) Physicomechanical characteristics of commercially available bulk-fill composites. J Dent 42: $993-1000$.

12. Massotti TG, Barcellos DC, Petrucelli N, Tribst JP, de Paiva Gonçalves SE (2015) Analysis of flexural strength of composite resins polymerized by 2nd and 3rd generation leds. Brazilian Dental Science 18: 67-74.

13. Rosa RS, Balbinot C, Blando E, Mota E, Oshima H, et al (2012) Evaluation of mechanical properties on three nanofilled composites. Stomatologija 14: 126-30.

14. Monteiro GQdM, Montes MAJR (2010) Evaluation of linear polymerization shrinkage, flexural strength and modulus of elasticity of dental composites. Mat Res 13: 51-5.

15. Kimmes NS (2013) Commentary: the effect of a modeling resin and thermocycling on the surface hardness, roughness, and color of different resin composites. J Esthet Restor Dent 25: 420-1. 
16. Tuncer S, Demirci M, Tiryaki M, Unlu N, Uysal O (2013) The effect of a modeling resin and thermocycling on the surface hardness, roughness, and color of different resin composites. J Esthet Restor Dent 25: 404-19.

17. Peampring C, Sanohkan S (2014) Effect of Thermocycling on Flexural Strength and Weibull Statistics of Machinable Glass-Ceramic and Composite Resin. J Indian Prosthodont Soc 14: 376-80.

18. Ito S, Hoshino T, Iijima M, Tsukamoto N, Pashley DH, Saito T (2010) Water sorption/solubility of self-etching dentin bonding agents. Dent Mater 26: 617-26.

19. Malacarne J, Carvalho RM, de Goes MF, Svizero N, et al. (2006) Water sorption/solubility of dental adhesive resins. Dent Mater 22: 973-80.

20. Calais JG, Soderholm KJ (1988) Influence of filler type and water exposure on flexural strength of experimental composite resins. J Dent Res 67: 836-40.

21. Ito S, Hashimoto M, Wadgaonkar B, Svizero N, Carvalho RM, et al. (2005) Effects of resin hydrophilicity on water sorption and changes in modulus of elasticity. Biomaterials 26: 6449-59.

22. Palin WM, Fleming GJ, Burke FJ, Marquis PM, Randall RC (2005) The influence of short and medium-term water immersion on the hydrolytic stability of novel lowshrink dental composites. Dent Mater 21: 852-63.

23. Standard I (2000) ISO 4049 polymer based filling, restorative and luting materials. International Organization for Standardization 1-27.

24. Akin H, Ozdemir AK (2013) Effect of corrosive environments and thermocycling on the attractive force of four types of dental magnetic attachments. Journal of Dental Sciences 8: 184-8.

25. do Amaral FL, Colucci V, de Souza-Gabriel AE, Chinelatti MA, Palma-Dibb RG, et al. (2008) Adhesion to Er:YAG laser-prepared dentin after long-term water storage and thermocycling. Operative dentistry 33: 51-8.

26. Curtis AR, Shortall AC, Marquis PM, Palin WM (2008) Water uptake and strength characteristics of a nanofilled resin-based composite. J Dent 36:186-93.

27. Martin N, Jedynakiewicz NM, Fisher AC (2003) Hygroscopic expansion and solubility of composite restoratives. Dental materials 19 : 77-86.

28. Soderholm KJ, Mukherjee R, Longmate J (1996) Filler leachability of composites stored in distilled water or artificial saliva. J Dent Res 75: 1692-9.

29. Ritter J, Grayeski W, Lardner T (1996) Cyclic fatigue-crack growth along polymer/glass interfaces. Polymer Engineering \& Science 36: $2382-8$.

30. Cesar PF, Miranda WG, Jr., Braga RR (2001) Influence of shade and storage time on the flexural strength, flexural modulus, and hardness of composites used for indirect restorations. J Prosthet Dent 86: 289-96.

31. Sonwane SR , Hambire UV (2015) Comparison of Flexural \& Compressive Strengths of Nano Hybrid Composites. Inter J Engin Trends Appli 2: 47-52.

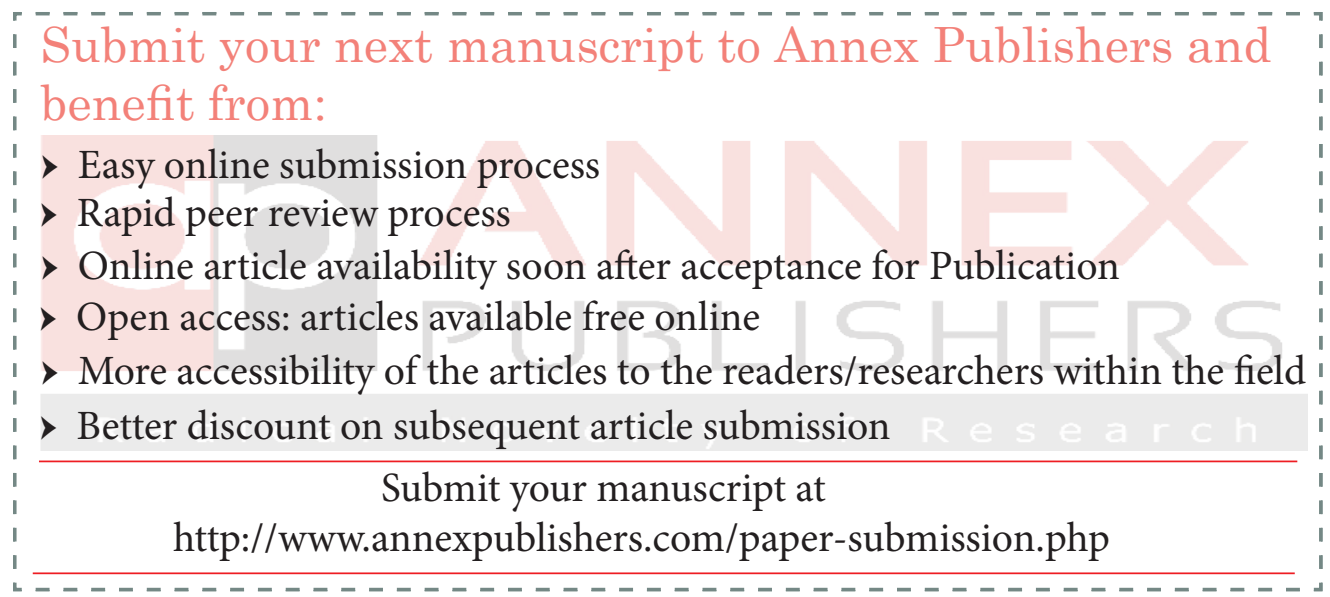

